\title{
MEASUREMENT OF ULTRA LOW OUTGASSING RATES FOR NLC UHV VACUUM CHAMBERS*
}

\author{
K. Kishiyama ${ }^{\dagger}$, S. Shen, D. Behne, Lawrence Livermore National Laboratory, Livermore, CA \\ 94550 \\ J.N. Corlett, D. Atkinson, K. Kennedy, T. Miller, Lawrence Berkeley National Laboratory, \\ Berkeley, CA, 94720 \\ L. Eriksson, M. Ross, Stanford Linear Accelerator Center, Stanford, CA, 94309
}

\begin{abstract}
Ultra low outgassing rates would be highly advantageous in accelerators and storage rings, such as the Next Linear Collider (NLC), where an outgassing rate of $<10^{-12}$ Torr liter/sec/ $\mathrm{cm}^{2}$ could eliminate the need for costly distributed pumping. Measuring such low outgassing rates at room temperature has many difficulties. However, by inspection of Fick's law, it can be seen that thermal desorption is proportional to outgassing rate. It is commonly observed that the outgas rate doubles approximately every $15^{\circ} \mathrm{C}$ for temperatures under $100^{\circ} \mathrm{C}$. By measuring outgassing rate versus temperature and time and extrapolating back to room temperature we can measure outgassing rates that would otherwise be difficult to make. To produce a reliable measurement also requires the total surface area under study to be approximately an order of magnitude greater than the area of the measurement chamber walls. To accomplish this, 27 plates of 5083 aluminum were placed in the measurement chamber. This technique will be the basis for future investigation of outgassing rates of other sample plates fabricated with different machining and cleaning techniques.
\end{abstract}

\section{INTRODUCTION}

The general technique used to measure outgassing rates is a chamber of volume $\mathrm{V}$ pumped through an orifice of known conductance, C. On each side of the orifice, gauges record the pressure. When certain conditions are met, the outgassing rate can be calculated as $\mathrm{Q}=\mathrm{C}\left(\mathrm{P}_{2}-\mathrm{P}_{1}\right)$. There are many difficulties for measuring ultra low outgassing rates with this technique[1].

A common problem is when the outgassing rate on the sample side of the chamber is very low, the differential pressure across the orifice is smaller than the accuracy of the gauges resulting in large measurement errors. If the outgassing rate of sample side of the chamber becomes even lower, the total adsorption of gas molecules on the surfaces is greater than the desorption and a negative differential pressure can result, in other words, the sample side is out pumping the orifice.

By inspection of Fick's law, it can be seen that thermal

\footnotetext{
* Work supported by the U.S. Department of Energy under Contract Nos. DE-AC03-76SF00515 (SLAC), DE-AC0376SF00098 (LBNL) and W-7405-ENG-48 (LLNL)

"kishiyama1@1lnl.gov
}

desorption is proportional to outgassing rate[2]. It is

$$
q=C_{0} \sqrt{\frac{D}{\pi t}}\left[1+2 \sum_{n=1}^{n=\infty}(-1)^{n} e^{-n^{2} \frac{l^{2}}{D t}}\right]
$$

commonly observed that the outgassing rate doubles approximately every $15^{\circ} \mathrm{C}$ for temperatures under $100^{\circ} \mathrm{C}$. Heating the measurement chamber produces higher outgassing rates from the sample which results in a greater differential pressure across the orifice allowing for a more accurate measurement. Using the elevated temperature data to extrapolate back to room temperature, outgassing rates can be measured that would otherwise be difficult to make.

\section{SYSTEM DESCRIPTION}

The LLNL outgassing measurement vessel is a rectangular chamber that is divided into two subchambers by a wall that has a fixed diameter orifice. The sub-chamber for the samples is a 6 inch cube with an 8 inch conflat on top for inserting samples. The primary instrumentation consists of two Granville Phillips Model 370 Stabil-ion gauges, one mounted on each side of the orifice.

Each 370 Stabil-ion gauge is individually calibrated (for nitrogen) at the factory and is shipped with a memory module containing the calibration curve. The memory module is then downloaded into the gauge controller and provides $3 \%$ accuracy.

The actively pumped side of the vessel uses a Seiko Seiki magnetically levitated turbomolecular pump and also has a Leybold-Inificon Transpector II RGA.

Thermocouple, ion gauge and RGA data is recorded by a G3 PowerMac running LabView software. The software also controls the temperature of the chamber through a temperature controller. The conductance of the orifice is corrected for temperature and the outgassing rate is calculated from the differential pressure across the orifice.

The outgassing rate of the chamber itself has been measured many times and is subtracted from the total measured outgassing rate to provide the outgassing rate of the sample. However, since all the parameters effecting the outgassing rate of the blank runs cannot be perfectly controlled, the chamber outgassing rate can vary. Also, during the heating cycle, the exact activation energy of desorption of the sample or the chamber walls are not 
known. Or in other words, one cannot assume the specific outgassing rates are equal when they are at the same temperature. Using a sample surface area 10 times greater than the chamber surface area helps minimize these errors.

\subsection{Sample Preparation}

Aluminum vacuum chambers are planned for NLC to save expense. It was decided to attempt to duplicate the results at ELETTRA[3], where they reported rates as low as $1 \times 10^{-13}$ Torr-liter $/ \mathrm{sec} / \mathrm{cm}^{2}$ from milled surfaces with a finish of $0.2 \mu \mathrm{m}$ RA.

Flat plates were chosen because they would be the most easily produced sample and would most likely achieve the desired surface finish. Additionally, they facilitate packing the most surface area into the measurement chamber volume. Plates of 5083-H321 were purchased in a thickness of $6.4 \mathrm{~mm}$ and cut into $145 \times 145 \mathrm{~mm}$ squares. They were held in a vacuum chuck in a horizontal milling machine and reduced to the final thickness of $3.2 \mathrm{~mm}$ using Trim ${ }^{\circledR}$ Sol cutting fluid that was sulfur and siliconefree. All six surfaces of each plate were machined. The final surface finish varied from a minimum of $0.2 \mu \mathrm{m}$ RA to a maximum of $0.3 \mu \mathrm{m} \mathrm{RA}$, with an average of about $0.24 \mu \mathrm{m}$ RA. The samples were then vapor degreased with perchlorethylene and rinsed with deionized water.

A comb shaped sample holder was inserted into the measurement chamber and held 27 sample plates. The total surface area of the sample plates was $11,473 \mathrm{~cm}^{2}$.

\section{MEASUREMENTS}

The sample plates and measurement chamber were initially conditioned and an outgassing rate measured at a steady state temperature. In the following runs, the measurement chamber heaters were stepped up $15^{\circ} \mathrm{C}$ every four hours and the outgassing rate measured as a function of temperature

\subsection{Initial Conditioning}

The sample plates and measurement chamber were initially conditioned by baking at $150^{\circ} \mathrm{C}$ for 24 hours. Future bakeout recipes will be adjusted to reflect the requirements of the metallurgy such that the heat treating will not be affected. After cooling down, the system was passivated for 30 minutes with nitrogen boil off from a liquid nitrogen tank and then pumped down for 120 hours.

\subsection{Temperature Step Measurements}

For these tests, the measurement chamber heaters were then stepped up $15^{\circ} \mathrm{C}$ every 4 hours. In addition, for the second and third runs the temperature was held at the final setpoint for 24 hours to simulate low temperature bakeouts. The ion gauge pressures and temperatures of the sample and vessel walls of the third run versus time is shown in the plot in Figure 1. The starting and ramping pressure and temperature profiles are typical of all three runs.
The heat radiated to the sample plates under vacuum from the hot filament ion gauge results in the initial steady state plate temperature of $52^{\circ} \mathrm{C}$. The initial chamber wall temperature is $30^{\circ} \mathrm{C}$ because of conduction and convection cooling with the atmosphere. Anytime there is one surface cooler than the other, it is hard to predict the interaction of the rates of adsorption or desorption between the two surfaces.

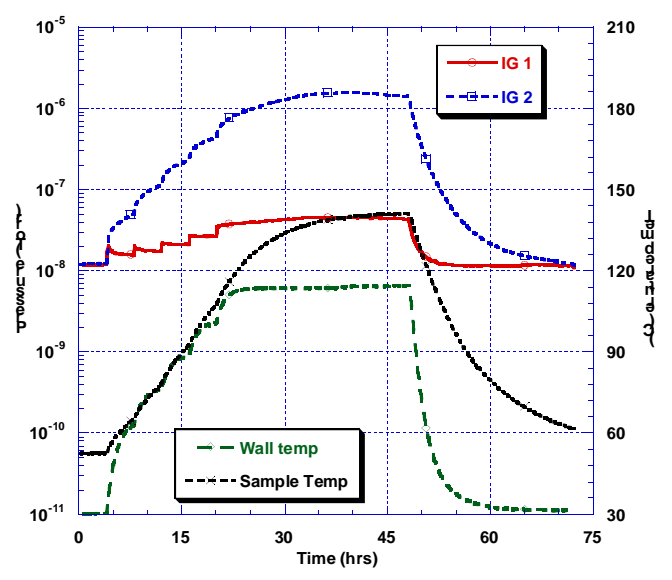

Figure 1. Pressure and temperature versus time

During the phase where the temperature was stepped up, the chamber wall and sample plate temperatures become equal. During this phase it is assumed that thermal desorption is the dominate term for both surfaces.

As noted before, Figure 1 shows the third run where the final setpoint temperature was held for 24 hours. Figure 1 shows that while the chamber wall temperature was held constant for the 24 hours, the sample plate temperature continued to increase. Since it is actually the measurement chamber wall temperature that is being controlled, the chamber wall heat loss to atmosphere requires extra heat to be applied to hold the wall temperature constant. The sample plate temperature rise is due to the heat loss of the chamber walls to atmosphere being greater than the heat loss of the sample plates due to re-radiation in vacuum. Because it is hard to predict the interaction of gas molecules between the two surfaces at different temperatures, it is difficult to determine the true outgassing rate of the sample during this phase.

The temperature steps also constitute baking out the system, so lower outgassing rates would be expected as the system cools down. However, during the cooldown cycle, the vessel walls cool at a faster rate than the sample plates, which results in a very low apparent outgassing rate. It would be very difficult in this phase to determine the true outgassing rate of the sample since we do not know the adsorption rate of the vessel walls versus the desorption rate of the sample plates. As further cooling occurs, the differential pressure across the orifice becomes very small and eventually becomes negative indicating 
that the adsorption by the surfaces is greater than the desorption and the conductance of the orifice.

Figure 2 is a plot showing three consecutive runs of outgassing rate versus temperature. The upper portion of each curve is the outgassing rate during the heating cycle. The lower portion of each curve is the cooldown cycle. After the cooldown cycle, the next run would be started without passivating the system. Note the very low apparent outgas rates measured in the cooldown curves.

The downward shift in the three curves shows the cleaning effect of baking out at low temperatures and long term pumping. Because of the clean surface conditions at the start of the second and third runs, the initial calculated outgassing rates appear to be very low. Once the temperature starts to increase, the thermal desorption increases and the measured outgas rate takes a large jump. At around $60^{\circ} \mathrm{C}$ in the heating cycle, as the temperatures of the vessel wall and sample plate equalize, the relationship between the $\log$ of the outgas rate and temperature becomes linear as predicted.

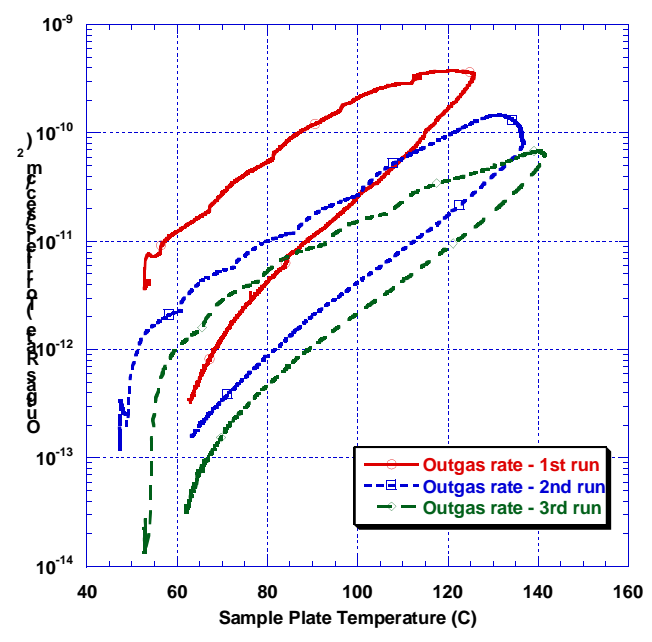

Figure 2. Outgas rate versus temperature for three runs

The start of the second and third runs also demonstrates the difficulty in very low outgassing measurements at lower temperatures, especially after a bakeout. Since the differential pressure is very small, the relative percent measurement error is very large. Any small variation in the various parameters (such as temperature in this case) that influences the outgas rate causes a change in the differential pressure which can result in a large change in the measurement.

In Figure 3, a simple linear extrapolation back to room temperature is shown for each of the three runs. All three extrapolations use the same slope. The cooling cycle is omitted because it is difficult to determine the true outgassing rate of the sample as discussed earlier.

The second and third runs would represent typical surface conditions after a low temperature bakeout. Based on the extrapolation of the second and third runs, we measure the specific outgassing rate to be between $2 \times 10^{-13}$ and $8 \times 10^{-14}$ Torr liters $/ \mathrm{sec}^{-} \mathrm{cm}^{2}$.

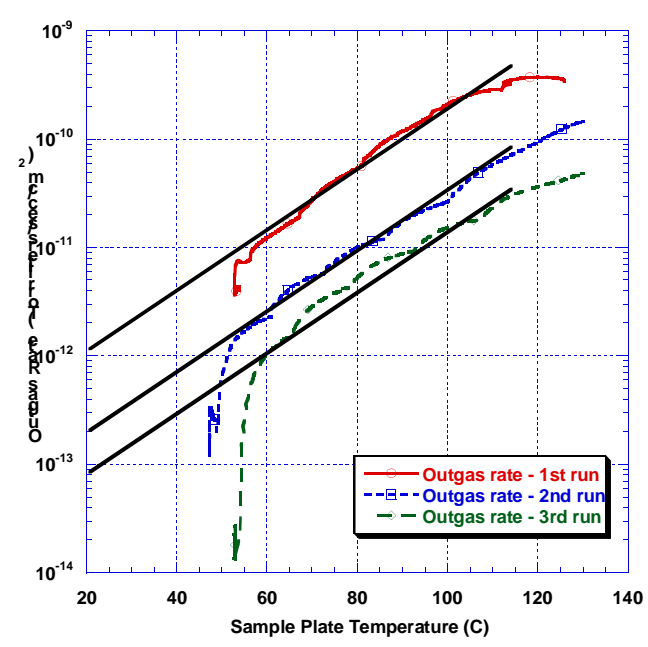

Figure 3. Outgassing rate extrapolation

By the end of the third run, the system had been under vacuum for over 500 hours and had been baked out to over $120^{\circ} \mathrm{C}$ four times (counting the initial conditioning run not shown in this report). It is predicted that the difference in the outgas versus temperature curves for additional runs would become smaller and smaller as the system cleans up even further.

\section{CONCLUSION}

The measurement technique presented here allows us to determine very low outgassing rates that could not be directly measured at room temperature, especially following a bakeout where the surfaces are very clean. In this technique, temperature data from the sample and the measurement chamber must be carefully examined to avoid using data that may not be valid. For higher confidence in the measurement, several cycles are needed to map the trend, which requires the sample to be under vacuum for several hundred hours.

\section{REFERENCES}

[1] Y. Strausser, Varian Technical Note VR-51

[2] D. Holkeboer, et. al. Vacuum Engineering, (Boston Technical Pub., Cambridge, Mass., 1967) p. 188

[3] A. Gambitta, et. al., "Experience With Aluminum Vacuum Chambers at ELETTRA," Proc. EPAC'00, p. 2292. 